\title{
Evolução Diferencial para eliminação de ruído em Analisadores de Espectro em Tempo Real baseados em MEMS *
}

\author{
Yara Quilles Marinho* Mateus Giesbrecht** \\ Fabiano Fruett ${ }^{* * *}$ \\ Faculdade de Engenharia Elétrica e de Computação, Universidade \\ Estadual de Campinas - UNICAMP. Campinas, SP. \\ *yaraqm@dsif.fee.unicamp.br \\ **mateus@fee.unicamp.br \\ ***fabiano@dsif.fee.unicamp.br
}

\begin{abstract}
The vibration spectrum, a tool used for monitoring various systems, is traditionally calculated by the Fast Fourier Transform (FFT) of a time series that represents the position of a mass subject to the forces that cause the vibration. Another possible alternative, with several operational advantages, is the use of microaccelerometers pairs to obtain the spectrum directly in the mechanical domain. This strategy has its greatest limitation on the accelerometers physical parameters differences - inherent to the production process -, such that the spectrum distortion level may be considerably higher than that found by the FFT. To overcome these differences, in this paper the adjustment of the microaccelerometers actuation voltages through a differential evolution algorithm for a multiobjective optimization based on the components of the discrete Fourier series of the spectrum analyzer system differential gain is proposed.

Resumo: O espectro de vibração, ferramenta utilizada para o monitoramento de sistemas diversos, é tradicionalmente calculado pela Transformada Rápida de Fourier (FFT) - do termo em inglês Fast Fourier Transform - de uma série temporal que representa a posição de uma massa sujeita às forças que causam a vibração. Outra alternativa viável, com diversas vantagens operacionais, é o uso de pares de microacelerômetros para a obtenção do espectro diretamente no domínio mecânico. Essa estratégia possui sua maior limitação nas diferenças nos parâmetros físicos dos acelerômetros - inerentes ao processo produtivo -, de tal forma que o nível de distorção do espectro pode ser consideravelmente superior àquele encontrado pela FFT. Para contornar essas diferenças, neste artigo é proposto o ajuste das tensões de atuação dos microacelerômetros através de um algoritmo de evolução diferencial para uma otimização multiobjetivo baseada nas componentes da série de Fourier discreta do ganho diferencial do sistema analisador de espectro.
\end{abstract}

Keywords: Accelerometers; Differential Evolution Algorithm; Multiobjective Optimization; MEMS.

Palavras-chaves: Acelerômetros; Algoritmo de Evolução Diferencial; Otimização Multiobjetivo; MEMS.

\section{INTRODUÇÃO}

A análise do espectro de vibração é uma ferramenta poderosa e bastante versátil, sendo usada em diversos sistemas de Engenharia. Atualmente, a solução mais utilizada para o levantamento do espectro de vibração é a análise no domínio elétrico, valendo-se de um acelerômetro para aquisição dos dados de uma série temporal e de um processador para a realização da Transformada Rápida de Fourier - FFT, do termo em inglês Fast Fourier Transform. O algoritmo para o cálculo da FFT pode ser implementado em um computador (Pedotti et al., 2017) ou em um proces-

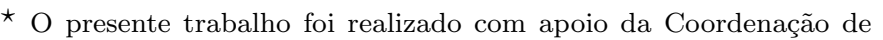
Aperfeiçoamento de Pessoal de Nível Superior - Brasil (CAPES) Código de Financiamento 001, e do Conselho Nacional de Desenvolvimento Científico e Tecnológico - Brasil (CNPq).
}

sador digital de sinais (Betta et al., 2002). Estas soluções apresentam sua principal desvantagem exatamente na necessidade do processador digital, que consome potência e cujas dimensões inviabilizam o uso em diversas aplicações, como, por exemplo, na área médica (Biefeld et al., 2000; Puers et al., 2000), em que é necessário o uso de microinstrumentação.

Com o desenvolvimento das tecnologias de fabricação do silício à partir da década de 1960 e o conhecimento de suas excelentes propriedades mecânicas, aliadas à versatilidade elétrica e térmica (Bao and Middelhoek, 2000), sistemas microeletromecânicos - também denominados MEMS, do termo em inglês Microelectromechanical Systems - popularizaram-se nas décadas seguintes, tornandose alternativas viáveis de sensores e atuadores em várias áreas. Na análise espectral, conforme observado em diver- 
sos trabalhos a partir do final da década de 1990 (Peiner et al., 1999), ao utilizar microacelerômetros, pode-se eliminar a necessidade de aplicação da FFT ao amplificar o sinal de vibração em uma pequena banda ao redor de sua frequência de ressonância (Scheibner et al., 2005).

Para contornar a restrição da frequência fixa dessa estratégia, pode ser empregada a realimentação de força eletrostática. Essa consiste em um mecanismo de sintonia da frequência de ressonância do micro-oscilador através da aplicação de uma tensão contínua na malha do dispositivo, permitindo a modulação eletrônica de sua constante de mola (Rocha et al., 2005). Essa sintonia possibilita a obtenção de diversas linhas espectrais simultaneamente ao se utilizar um arranjo de osciladores, cada um sintonizado em uma frequência específica (Lee and Cho, 1998; Ye et al., 1998; Scheibner et al., 2005).

Estendendo-se o conceito de realimentação de força eletrostática, um microacelerômetro pode ser seletivamente sensível a uma componente de frequência estreita da vibração mecânica através da aplicação de um sinal elétrico alternado na malha do dispositivo. Com isso, as componentes da vibração são obtidas sequencialmente pela variação da frequência do sinal de acionamento. Dessa forma, pode ser realizada uma filtragem espectral eletronicamente controlada no domínio mecânico. Essa é a proposta de Rocha et al. (2005), em que se utilizam dois microacelerômetros capacitivos em uma configuração diferencial para a obtenção do espectro de vibração, em tempo real, diretamente no domínio da frequência. Os autores apontam performance comparável com a FFT de uma série temporal. A parte crítica, entretanto, é a falta de correspondência completa dos parâmetros físicos dos dois acelerômetros usados na estratégia - tais como massas e constantes de mola -, uma vez que qualquer diferença entre esses parâmetros leva ao aparecimento de linhas espectrais inexistentes no sinal de vibração monitorado.

Para contornar essas diferenças e eliminar o ruído do espectro de vibração, propõe-se no presente artigo uma abordagem de calibração do analisador de espectro através da amplitude das tensões de excitação dos acelerômetros. Para tanto, o algoritmo de evolução diferencial foi adaptado para a realização de uma otimização multiobjetivo, de forma a determinar uma combinação de tensões de excitação dos microacelerômetros para maximizar a componente harmônica de primeira ordem do ganho diferencial do sistema ao mesmo tempo em que se minimiza a soma das demais componentes. Os meta-parâmetros da evolução diferencial foram escolhidos através de uma busca exaustiva, considerando-se não somente o tempo de convergência, mas também os piores e melhores casos obtidos com a evolução diferencial. As calibrações realizadas têm suas características, vantagens e desvantagens explicitadas; essas calibrações são testadas ao submeter o sistema analisador de espectro a um sinal de aceleração distinto daquele utilizado para a calibração.

Este artigo é dividido da seguinte maneira: na seção 2, um pequeno histórico dos sistemas microeletromecânicos é apresentado, justificando as vantagens de sua utilização no analisador de espectro em tempo real, cujo funcionamento básico é descrito em seguida, na seção 3. Uma breve introdução sobre os problemas de otimização é apresentada na seção 4, bem como das meta-heurísticas para resolvê-los quando sua complexidade é elevada. Nessa mesma seção, o algoritmo de evolução diferencial é descrito em detalhes, além de ser apresentada a possibilidade de sua utilização para problemas de otimização multiobjetivo. A seção 5 trata dos resultados obtidos para a simulação do analisador de espectro e para a calibração dos pares de acelerômetros, validando sua implementação. Por fim, na seção 6, são apresentadas as conclusões do artigo.

\section{SISTEMAS MICROELETROMECÂNICOS}

O desenvolvimento de microssistemas resulta dos progressos da microeletrônica desde a década de 1960, mas foi a partir de 1980 que os maiores avanços foram conquistados (Senturia, 2001). Sistemas microeletromecânicos envolvem elementos eletrônicos e não-eletrônicos e são definidos como a integração de elementos mecânicos, sensores, atuadores e a eletrônica para processamento de sinal em um substrato comum de silício através da tecnologia de microfabricação (Younis, 2011). Esta tecnologia permite a produção estável e confiável de uma quantidade numerosa de dispositivos ao mesmo tempo, de modo que a produção dos MEMS em grande escala se dá a um custo relativamente baixo (Senturia, 2001; Younis, 2011). Somam-se a isso seu baixo peso, tamanho reduzido e baixo consumo de energia (Beeby et al., 2006), além de sua performance superior, suas funcionalidades inteligentes e a possibilidade de realizar tarefas complexas que não seriam resolvidas com outras tecnologias (Younis, 2011).

Uma categoria de MEMS amplamente utilizada em diversas aplicações é a dos sensores inerciais - também chamados acelerômetros -, que podem ser projetados e fabricados para serem sensíveis a componentes de aceleração em um, dois ou três eixos. A aceleração pode ser convertida em um sinal elétrico através do uso de transdutores capacitivos (Tez et al., 2016), piezoresistivos (Roy and Bhattacharyya, 2015), indutivos (Tavakkoli et al., 2017), entre outros. Entretanto, acelerômetros capacitivos - que podem apresentar topologias variadas, como massas suspensas por um único suporte ou por vários (van Kampen and Wolffenbuttel, 1998) - são os mais populares, pois possuem diversas vantagens sobre os macrossistemas, entre elas: alta sensibilidade e baixo nível de ruído, associadas com baixo consumo de energia, custo reduzido e baixa dependência com a temperatura.

\section{ANALISADOR DE ESPECTRO EM TEMPO REAL BASEADO EM MEMS}

A operação do dispositivo proposto por Rocha et al. (2005), baseado em microacelerômetros capacitivos com massa engastada em uma extremidade, pode ser compreendida tomando como base um pêndulo invertido convencional, engastado em uma extremidade, inserido em um campo gravitacional vertical $\vec{g}$, conforme mostrado na Figura 1. A massa sísmica $m$ é suposta como concentrada no topo, enquanto a viga engastada tem apenas propriedades elásticas, desconsiderando-se sua massa. Qualquer força horizontal externa $F_{x, \text { ext }}$ aplicada ao sistema causa um deslocamento $\varphi$ a partir da posição vertical de equilíbrio, até que a reação desenvolvida no ponto de engaste equilibre a ação externa. Sem um campo gravitacional, essa ação 
externa é unicamente determinada pela força horizontal externa; entretanto, na presença de um campo gravitacional, o efeito é ampliado pela componente tangencial da força peso, $F_{v, t}$, e, assim, resulta em uma maior deflexão de equilíbrio. A componente tangencial da força peso, portanto, amplifica o efeito rotacional da força externa, induzindo uma realimentação positiva.

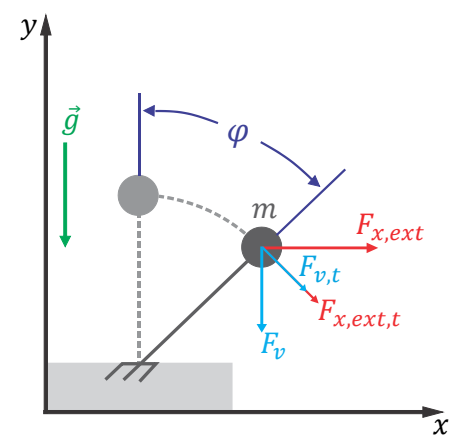

Figura 1. Princípio de operação do pêndulo invertido no campo gravitacional.

Em microssistemas, o mesmo princípio é válido ao substituir o campo gravitacional vertical por um campo eletrostático, com a grande vantagem da possibilidade de controle eletrônico deste campo. Assim, há a possibilidade de ajuste dinâmico do efeito de realimentação, com a sintonia da sensibilidade e da seletividade espectral com respeito à entrada de aceleração horizontal (Cretu et al., 1999; Rocha et al., 2005).

O modelo do acelerômetro MEMS, desenvolvido analiticamente por Cretu et al. (1999), é mostrado esquematicamente na Figura 2, no caso do modo de operação quaseestático, que é aquele em que a frequência de atuação é mais baixa que a frequência de ressonância mecânica. Nessa figura, $a_{\text {ext }}$ representa a aceleração causada pela força $F_{x, \text { ext }}$ sobre a massa $m, k$ é a constante elástica da mola mecânica do dispositivo, $\Delta k$ é a alteração na constante elástica da mola causada pela realimentação, $v(t)$ é a tensão de atuação do microacelerômetro, $V_{0}$ é a amplitude da tensão e $x(t)$ é o sinal de saída do acelerômetro.

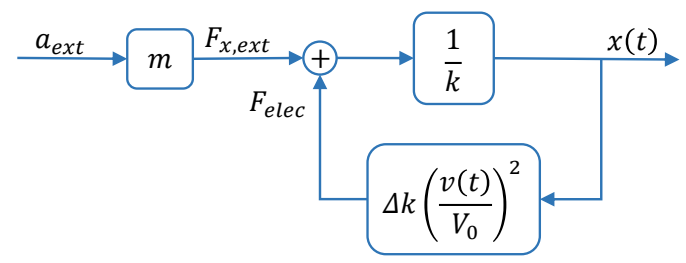

Figura 2. Modelo quase-estático do acelerômetro MEMS.

Nota-que que a tensão de atuação $v(t)$ - que define o ganho e o ponto de operação - além de ser quadrática, está presente não no caminho direto, mas na realimentação. A partir da Figura 2 é possível escrever a Equação (1) para o sinal de saída do acelerômetro MEMS.

$$
\begin{aligned}
x(t) & =F_{x, e x t} \frac{1}{k-\Delta k\left(\frac{v(t)}{V_{0}}\right)^{2}} \\
& =\frac{m a_{e x t}}{k} \frac{1}{1-\beta\left(\frac{v(t)}{V_{0}}\right)^{2}},
\end{aligned}
$$

sendo que $\beta=\Delta k / k$ é a constante de modulação adimensional, que depende do projeto e das características da malha de realimentação.

Com uma tensão de excitação do campo elétrico $v(t)$ variante no tempo, ocorre um uso mais avançado do acoplamento estrutural entre os campos mecânico e eletrostático, que é a proposta de Rocha et al. (2005). Conforme mostrado na Figura 3, uma estrutura de acelerômetros gêmeos, com acionamento em quadratura, é utilizada.

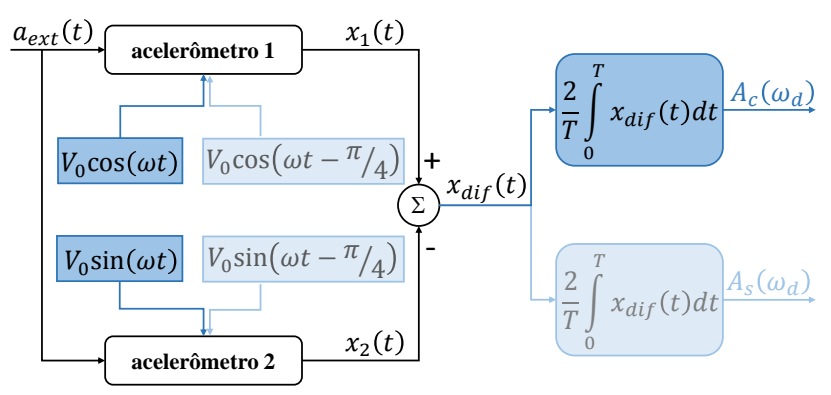

Figura 3. Diagrama de blocos da estrutura diferencial para a obtenção do espectro mecânico.

No caso de uma tensão de atuação cossenoidal, $v_{1}(t)=$ $V_{0} \cos (\omega t)$, aplicada ao acelerômetro 1 da estrutura, de massa $m_{1}$, constante elástica $k_{1}$ e constante de modulação $\beta_{1}$, é possível obter a Equação (2) para o sinal de saída:

$$
\begin{aligned}
x_{1}(t) & =\frac{m_{1}}{k_{1}} \frac{1}{1-\beta_{1}\left(\frac{V_{0} \cos (\omega t)}{V_{0}}\right)^{2}} a_{e x t}(t) \\
& =\frac{m_{1}}{k_{1}} \frac{1}{1-\frac{\beta_{1}}{2}-\frac{\beta_{1}}{2} \cos (2 \omega t)} a_{e x t}(t) .
\end{aligned}
$$

Já o acelerômetro 2 , de massa $m_{2}$, constante elástica $k_{2}$ e constante de modulação $\beta_{2}$, possui tensão de excitação $v_{2}(t)=V_{0} \sin (\omega t)$. Assim, o sinal de saída no tempo do segundo acelerômetro é dado pela Equação (3).

$$
\begin{aligned}
x_{2}(t) & =\frac{m_{2}}{k_{2}} \frac{1}{1-\beta_{2}\left(\frac{V_{0} \sin (\omega t)}{V_{0}}\right)^{2}} a_{e x t}(t) \\
& =\frac{m_{2}}{k_{2}} \frac{1}{1-\frac{\beta_{2}}{2}+\frac{\beta_{2}}{2} \cos (2 \omega t)} a_{\text {ext }}(t)
\end{aligned}
$$

Dessa forma, após a subtração dos sinais de saída dos dois acelerômetros, a saída diferencial variante no tempo é dada pela Equação (4) - quando os acelerômetros são idênticos e todos os parâmetros físicos correspondem aos respectivos valores nominais.

$$
x_{d i f}(t)=\frac{m \beta \cos (2 \omega t)}{k\left(1-\beta+\frac{\beta^{2}}{4}-\frac{\beta^{2}}{4} \cos ^{2}(2 \omega t)\right)} a_{e x t}(t)
$$

Assim, a saída diferencial contém um sinal proporcional a $a_{\text {ext }}(t) \cos (2 \omega t)$, onde $a_{\text {ext }}$ é a entrada de aceleração, enquanto $\omega$ é a frequência da força eletrostática de acionamento. 
O formato da Equação (4) sugere a possibilidade de aplicar um método de correlação, de modo a extrair a componente espectral desejada $A\left(\omega_{d}\right)$ do sinal de aceleração mecânica de entrada em uma determinada frequência $\omega_{d}$, conforme a Equação (5).

$$
\begin{aligned}
A\left(\omega_{d}\right) & =\lim _{T \rightarrow \infty} \frac{2}{T} \int_{0}^{T} a_{e x t}(t) e^{-j \omega_{d} t} d t \\
& =\lim _{T \rightarrow \infty} \frac{2}{T} \int_{0}^{T} a_{e x t}(t)\left[\cos \left(\omega_{d} t\right)-j \sin \left(\omega_{d} t\right)\right] d t \\
& =A_{c}\left(\omega_{d}\right)-j A_{s}\left(\omega_{d}\right),
\end{aligned}
$$

em que $A_{c}\left(\omega_{d}\right)$ e $A_{s}\left(\omega_{d}\right)$ correspondem, respectivamente, à parcela cossenoidal e à parcela senoidal da componente espectral $A\left(\omega_{d}\right)$ da aceleração externa.

Dessa forma, conforme indica a Equação (5), é possível obter $A_{c}(2 \omega)$ através da integração do sinal de saída diferencial $x_{\text {dif }}(t)$. Já para a obtenção de $A_{s}(2 \omega)$, uma atuação similar em quadratura, mas como uma diferença de fase de $\pi / 4$ é utilizada, gerando uma saída proporcional $a_{\text {ext }}(t) \sin (2 \omega t)$. Assim, como a diferença de fase entre a aceleração externa $a_{\text {ext }}(t)$ e as tensões de excitação dos acelerômetros são desconhecidas, a amplitude $|A(2 \omega)|$ da componente espectral de frequência $2 \omega$ pode ser calculada através do uso da Equação (6):

$$
|A(2 \omega)|=\sqrt{A_{c}^{2}(2 \omega)+A_{s}^{2}(2 \omega)} .
$$

A estratégia descrita pode ser implementada utilizando-se um método de multiplexação temporal, como mostrado na Figura 3. Para isso, ora são utilizadas as tensões de atuação em tons mais fortes, obtendo-se a componente cossenoidal de $A(2 \omega)$ após a integração do sinal de saída diferencial; ora são aplicadas as tensões em tons mais claros, obtendose a componente senoidal de $A(2 \omega)$ com a integração do sinal de saída. Assim, ao variar a frequência $\omega$ da atuação elétrica, o espectro de vibração mecânica é mapeado.

Os resultados obtidos por Rocha et al. (2005) com o mapeamento do espectro em tempo real comparam-se bem com a FFT para o mesmo tempo de aquisição. Similarmente à abordagem FFT, a seletividade do mapeamento do espectro em tempo real depende do tempo de integração ou aquisição.

\subsection{Espectro e Distorções}

Conforme a Equação (5), o método de correlação para a obtenção da componente espectral $A\left(\omega_{d}\right)$ ocorre de maneira eficiente quando o sinal de aceleração externa é multiplicado por sinais cossenoidais e senoidas - para a obtenção de $A_{c}\left(\omega_{d}\right)$ e $A_{s}\left(\omega_{d}\right)$, respectivamente. No caso do sistema analisador de espectro utilizado, no entanto, os sinais que multiplicam a aceleração externa não são cossenoides e senoides puras, conforme mostrado na Equação (4) e, para avaliar seu efeito no levantamento do espectro, pode-se analisar a Equação (7), que representa o ganho diferencial do sistema para o intervalo em que a componente $A_{c}(2 \omega)$ é computada.

$$
G_{d i f}(t)=\frac{x_{d i f}(t)}{a_{e x t}(t)}=\frac{m \beta \cos (2 \omega t)}{k\left(1-\beta+\frac{\beta^{2}}{4}-\frac{\beta^{2}}{4} \cos ^{2}(2 \omega t)\right)}
$$

Como o ganho $G_{d i f}(t)$ é periódico, é possível realizar uma decomposição em série de Fourier discreta. Devido às propriedades de simetria, apenas os coeficientes cossenoidais ímpares aparecem na série de Fourier. Dessa forma, no caso ideal de acelerômetros idênticos, apesar de o ganho não ser puramente cossenoidal, pelo fato de haver um termo cossenoidal no denominador da equação, este é pouco significativo e, nos casos em que $\beta<1$, pouco interfere no espectro obtido com o acelerômetro.

Em casos reais, entretanto, o nível de ruído é maior na abordagem proposta, uma vez que os parâmetros físicos dos acelerômetros apresentam diferenças entre si. Assim, a Equação (7) não representa fielmente o ganho do sistema diferencial e, com isso, a série de Fourier discreta do ganho diferencial acaba por apresentar outras componentes além das cossenoidais ímpares e, consequentemente, o espectro obtido pela aplicação da estratégia com os acelerômetros MEMS reais mostra frequências inexistentes no espectro de entrada real.

Para a eliminação das componentes indesejadas do espectro, as diferenças entre os parâmetros dos acelerômetros podem ser compensadas ajustando-se sua tensão de excitação, de forma a alterar suas constantes de modulação adimensionais $\beta_{1}$ e $\beta_{2}$. Para isso, propõe-se utilizar acelerações de teste conhecidas e o sinal de saída diferencial do sistema para a análise do ganho diferencial $G_{\text {dif }}(t)=x_{\text {dif }}(t) / a_{\text {ext }}(t)$, tornando-o mais próximo de um ganho senoidal ou cossenoidal - dependendo do intervalo de tempo considerado, uma vez que a estratégia da Figura 3 envolve uma multiplexação temporal para a obtenção das parcelas senoidal e cossenoidal do espectro. Dessa forma, é importante atentar-se para a definição dos intervalos de tempo a serem utilizados para o cálculo da série de Fourier: além da multiplexação temporal, como o espectro é obtido através da variação da frequência $\omega$ da tensão de atuação, é importante que o intervalo considere apenas uma dessas frequências de atuação. Assim, ao maximizar a componente fundamental da série de Fourier do ganho diferencial nesse intervalo de tempo, ao mesmo tempo em que as demais componentes são minimizadas, podem-se compensar as diferenças dos parâmetros do par de acelerômetros, tornando a resposta do sistema real mais próxima da resposta ideal.

Essa estratégia pode ser considerada como uma calibração inicial do par de acelerômetros que constitui o sistema analisador de espectro: em ambiente controlado, submetendose os acelerômetros a acelerações de teste conhecidas, são definidas as tensões de acionamento a serem utilizadas posteriormente sob condições desconhecidas de aceleração para eliminação do ruído presente no espectro final.

Para a determinação das diferentes tensões de excitação que maximizam o valor da fundamental e minimizam as harmônicas indesejadas, deve ser resolvido um problema de otimização o que, neste artigo, é realizado com técnicas meta-heurísticas, conforme detalhado na próxima seção.

\section{OTIMIZAÇÃO E META-HEURÍSTICAS}

O conceito de otimização pode ser entendido como a procura pela melhor solução possível dentre aquelas disponíveis (Boussaïd et al., 2013). Embora existam diversos 
métodos determinísticos de otimização, alguns problemas apresentam dificuldade elevada. Nesses casos, diversos algoritmos de busca, tais como as meta-heurísticas, podem ser utilizados para encontrar uma solução do problema. Dentre algumas das meta-heurísticas mais utilizadas estão simulated annealing, busca tabu e algoritmos genéticos (Gendreau and Potvin, 2010), sendo que estes últimos englobam diversos algoritmos - como a evolução diferencial, descrita a seguir.

\subsection{Evolução Diferencial}

A evolução diferencial - DE, do termo em inglês Differential Evolution - é um dos algoritmos mais populares para a otimização global de problemas contínuos (Boussaïd et al., 2013). Proposta por Storn and Price (1997), na evolução diferencial, como em todos os outros algoritmos evolutivos, uma população inicial de soluções candidatas é arbitrariamente escolhida. Para cada geração do processo evolutivo, novos indivíduos são criados através do processo reprodutivo, que consiste em mutação e cruzamento. Cada membro da população atual compete com um novo indivíduo gerado pelo processo reprodutivo, de forma que apenas o que produz a solução mais adequada para o problema em questão é mantido na população seguinte. A evolução diferencial apresenta algumas variações (Price et al., 2005) que podem ser nomeadas através do código $D E / x / y / z$, onde $D E$ denota a evolução diferencial; $x$ indica o indivíduo base, podendo ser aleatório (rand) ou o melhor dentro da população (best); já $y$ indica o número de vetores diferença usados para a perturbação do vetor base; o esquema de cruzamento é indicado por $z$, podendo ser binomial (bin) ou exponencial (exp). A versão clássica do algoritmo de evolução diferencial, e também a mais usada, é denotada por $D E /$ rand $/ 1 /$ bin.

A evolução diferencial é um método de busca paralelo direto que utiliza $N$ vetores de dimensão $D$ como população em cada geração, sendo estes vetores representados por $\boldsymbol{x}_{i, g}-\operatorname{com} i=1,2,3, \ldots, N$. Na versão $D E / \mathrm{rand} / 1 / \mathrm{bin}$, para cada vetor alvo $\boldsymbol{x}_{i, g}$, a mutação gera um vetor de mutante $\boldsymbol{v}_{i, g}$ conforme a Equação (8).

$$
\boldsymbol{v}_{i, g+1}=\boldsymbol{x}_{r_{1}, g}+F\left(\boldsymbol{x}_{r_{2}, g}-\boldsymbol{x}_{r_{3}, g}\right) \text {, }
$$

onde $g$ denota a geração atual e os índices $r_{1}, r_{2}$ e $r_{3}$ são mutuamente diferentes e escolhidos aleatoriamente entre $[1, N]$. O fator de escala $F$ é uma constante real no intervalo $[0,2]$ que controla a amplificação da variação diferencial.

Além da mutação, para elevar a diversidade dos vetores perturbados, uma operação de cruzamento é introduzida, formando o vetor de teste

$$
\boldsymbol{u}_{\boldsymbol{i}, \boldsymbol{g}+\mathbf{1}}=\left(u_{1 i, g+1}, u_{2 i, g+1}, \ldots, u_{D i, g+1}\right)
$$

à partir da Equação (9):

$$
u_{j i, g+1}= \begin{cases}v_{j i, g+1} & \text { se } \operatorname{randb}(j) \leq C R \text { ou } j=\operatorname{rnbr}(i) \\ x_{j i, g} & \text { if } \operatorname{randb}(j)>C R \text { ou } j \neq \operatorname{rnbr}(i)\end{cases}
$$

com $j=1,2, \ldots, D$, onde $\operatorname{randb}(j)$ é a $j$-ésima avaliação do gerador de números aleatórios uniformemente distribuídos no intervalo $[0,1]$. O parâmetro $C R$ é chamado de constante de cruzamento e representa a probabilidade de criar elementos para o vetor de testes à partir do vetor mutante (Boussaïd et al., 2013). O índice $r n b r(i)$ é aleatoriamente escolhido entre $1,2, \ldots, D$, o que garante que o vetor de teste $\boldsymbol{u}_{i, g+1}$ contenha pelo menos uma componente do vetor mutante $\boldsymbol{v}_{i, g+1}$.

A etapa seguinte à mutação e ao cruzamento é a seleção. Nesta, para decidir se o vetor de teste $\boldsymbol{u}_{i, g+1}$ deve se tornar um dos indivíduos da população $G+1$, ele é comparado com o vetor alvo $\boldsymbol{x}_{i, g}$. No caso de um problema de minimização, se $\boldsymbol{u}_{i, g+1}$ leva a menor valor da função custo que $\boldsymbol{x}_{i, g}$, então o indivíduo $i$ da geração $G+1$ é $\boldsymbol{x}_{i, g+1}=\boldsymbol{u}_{i, g+1}$; caso contrário, o valor anterior é mantido, de forma que $\boldsymbol{x}_{i, g+1}=\boldsymbol{x}_{i, g}$.

Uma das grandes vantagens da evolução diferencial consiste em seus poucos meta-parâmetros de controle de busca: o tamanho da população $N$, a contante de diferenciação $F$ e o parâmetro de controle da taxa de cruzamento $C R$ (Boussaïd et al., 2013). Embora não seja possível determinar a priori esses meta-parâmetros, diversas metodologias foram propostas para essa finalidade; em Kerr and Mullen (2019), por exemplo, realiza-se uma busca exaustiva em grade, variando os parâmetros e analisando os resultados em termos de tempo médio, melhores e piores respostas encontradas. Este método também foi adotado neste artigo.

\subsection{Otimização Multiobjetivo}

Como o nome sugere, problemas multiobjetivos - os problemas de otimização mais frequentes em sistemas reais envolvem a otimização de múltiplos objetivos simultaneamente e que, frequentemente, encontram-se em conflito entre si. Isso resulta em um grupo de soluções alternativas - chamado de conjunto Pareto-ótimo - que devem ser consideradas equivalentes na ausência de informações sobre a relevância de cada objetivo. Por esse motivo, algoritmos evolutivos são uma opção natural para solução de problemas de otimização multiobjetivo, já que lidam com grupos de soluções candidatas. A evolução diferencial é, atualmente, uma das heurísticas mais populares para a resolução de problemas de otimização mono-objetivo em espaços de busca contínuos. Devido a esse sucesso, diversos autores propuseram seu uso para problemas de otimização multiobjetivo, conforme mostra a revisão realizada por Mezura-Montes et al. (2008). Nesta, os autores propõem uma classificação das adaptações da evolução diferencial para problemas multiobjetivo com relação ao uso do conceito de Pareto otimalidade. Uma interpretação simples desse conceito - para o caso de minimização de todas as funções custo - indica que uma solução $a$ é melhor que uma solução $b$ se todas as funções custo em $a$ forem menores ou iguais que aquelas de $b$, sendo que pelo menos uma das funções custo deve ser menor em $a$ do que em $b$ (Boyd and Vandenberghe, 2004).

\section{SIMULAÇ̃̃O DO ANALISADOR DE ESPECTRO E EVOLUÇÃO DIFERENCIAL PARA ELIMINAÇÃO DE RUÍDO}

Para a simulação do circuito analisador de espectro em tempo real, optou-se pelo uso do software Matlab para a construção do modelo dos acelerômetros, conforme as Equações (2) e (3) e a estratégia de multiplexação ilustrada na Figura 3. Foram utilizados os parâmetros físicos do 
acelerômetro descrito em Cretu et al. (2003) e optou-se por um sinal de aceleração externa de teste com componentes em toda a faixa de frequência considerada pelos autores, com amplitudes $30 \mathrm{~m} / \mathrm{s}^{2}$ em $53 \mathrm{~Hz}, 45 \mathrm{~m} / \mathrm{s}^{2}$ em $127 \mathrm{~Hz}, 9$ $\mathrm{m} / \mathrm{s}^{2}$ em $255 \mathrm{~Hz}$ e $21 \mathrm{~m} / \mathrm{s}^{2}$ em $432 \mathrm{~Hz}$. O analisador de espectro foi empregado com espaçamento de $1 \mathrm{~Hz}$ entre cada ponto do espectro, por período de integração de $2 \mathrm{~s}$ para cada um desses, obtendo-se o resultado da Figura 4(a) para o caso ideal - com acelerômetros idênticos. Este resultado valida a simulação do analisador de espectro MEMS, uma vez que esta foi capaz de reproduzir as componentes espectrais, mantendo as frequências e relações de amplitude adequadas. Para efeito de visualização, as amplitudes dos espectros da Figura 4 encontram-se normalizadas.

Após essa validação da simulação do sistema analisador de espectro, partiu-se para a simulação de casos reais, com diferenças nos parâmetros dos acelerômetros. Para isso, os parâmetros físicos $m, k, C_{d 0}$ e $d_{0}$ de ambos os acelerômetros do par foram variados aleatoriamente entre $80 \%$ e $120 \%$ de seu valor nominal. Os parâmetros $C_{d 0}$ e $d_{0}$ representam, respectivamente, a capacitância na condição de equilíbrio e a distância entre os eletrodos dos capacitores do acelerômetro MEMS; estes parâmetros são usados no cálculo de $\Delta k$ (Cretu et al., 2003). Na Figura 4(b) é mostrado um exemplo de como o espectro de vibração fica poluído quando os acelerômetros não são idênticos.
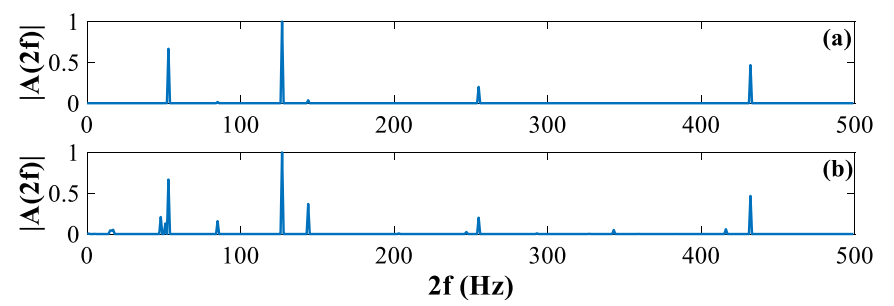

Figura 4. Espectros normalizados obtidos pela simulação do analisador MEMS - caso ideal(a) e um caso real (b).

Para entender a causa de tais distorções, pode-se recorrer ao ganho diferencial do sistema no caso ideal e no caso real, mostrados na Figura 5, juntamente com os respectivos coeficientes da série de Fourier discreta. Conforme anteriormente exposto, qualquer frequência de excitação - dentro da discretização do espectro proposta - pode ser escolhida para essa análise. Nesse caso, optou-se pela frequência de $1 \mathrm{~Hz}$ para facilitar a visualização e, portanto, o sinal de ganho ideal possui frequência de $2 \mathrm{~Hz}$ - devido à característica do analisador de espectro de obter a componente com dobro da frequência de excitação, demonstrada na Equação (6). Fica claro que, no intervalo de tempo proposto - referente ao cômputo da parcela cossenoidal do espectro -, o uso dos acelerômetros idênticos resulta em ganho diferencial muito próximo do ideal, o que é confirmado através da série de Fourier - que apresenta apenas o coeficiente de ordem 3, com pequena amplitude, além da componente fundamental. Já para o caso real, com diferenças entre os acelerômetros, o ganho diferencial apresenta-se consideravelmente distorcido em relação à cossenoide de referência, fato evidenciado pela composição da série de Fourier que apresenta coeficientes não-nulos de ordem 0 até 4 , justificando o aparecimento de componentes ruidosas no espectro mostrado na Figura 4(b).
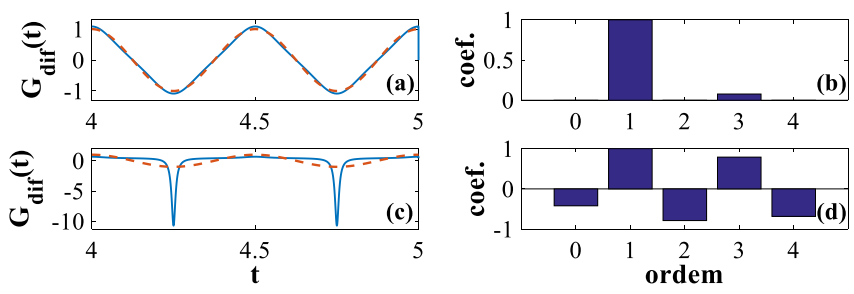

Figura 5. Ganho diferencial do analisador de espectro MEMS para componente cossenoidal (linha contínua) e cosseno ideal (linha pontilhada) - caso ideal (a) e um caso real (c). Coeficientes da série de Fourier do ganho diferencial - caso ideal (b) e um caso real (d).

Para contornar esse problema, conforme anteriormente citado, optou-se pela determinação das tensões de atuação que implicam na maximização da componente fundamental da série de Fourier do ganho diferencial do sistema associada à minimização da soma das demais componentes. Para isso, foi utilizado um sinal de aceleração externa com componente de $8,54 \mathrm{~m} / \mathrm{s}^{2}$ em $100 \mathrm{~Hz}$ e a série de Fourier do ganho diferencial foi computada no intervalo em que a tensão de excitação possui frequência de $1 \mathrm{~Hz}$ e a parcela cossenoidal da componente espectral é considerada. Novamente, a frequência de $1 \mathrm{~Hz}$ foi escolhida para facilitar a visualização durante a realização dos testes. O critério de parada escolhido para o algoritmo de evolução diferencial foi a primeira componente superior a 0,9 enquanto a soma das demais deveria ser inferior a 0,2 - considerando-se componentes normalizadas. Além disso, na seleção, o conceito de Pareto otimalidade foi utilizado, ou seja, caso uma das funções custo for melhor e a outra não for pior que a do indivíduo anterior, este é substituído. O critério de parada também inclui o número máximo de gerações - para evitar que o algoritmo continue indefinidamente nos casos em que as condições das componentes não possam ser satisfeitas e o desvio padrão dentre os indivíduos da geração - para garantir a diversidade da população. Como o algoritmo DE não é determinístico, optou-se por realizar cinco testes para cada par de acelerômetros. Dentre estes testes, os parâmetros de otimização correspondentes ao melhor caso foram selecionados para a calibração do dispositivo. O melhor caso corresponde àquele, dentre todos os testes, que garante o maior valor para a componente fundamental aliado à menor soma das demais componentes da série da Fourier.

Para a implementação da evolução diferencial para eliminação do ruído valendo-se das amplitudes das tensões $V_{1} \mathrm{e}$ $V_{2}$ de atuação doas acelerômetros, os parâmetros $N$ e $F$ do algoritmo de evolução diferencial foram escolhidos através de uma busca exaustiva em grade. Nessa, um mesmo par de acelerômetros foi utilizado para todas as buscas e foram avaliados os resultados de tempo médio, número médio de gerações, bem como melhor e pior resultados para as componentes da série de Fourier. Os gráficos foram aqui omitidos por simplicidade. Observou-se que os resultados que mais variavam eram o pior caso da soma das componentes e o tempo médio. Por isso, os parâmetros foram escolhidos de forma que o pior caso se encontrasse dentro do critério de parada - estabelecido anteriormente - e cujo 
tempo fosse o menor possível. Com isso, foram definidos $N=10$ e $F=1,3$. Cabe ressaltar que o parâmetro $C R$ foi mantido unitário, uma vez que as variáveis de otimização $V_{1}$ e $V_{2}$ são escalares e, portanto, o cruzamento deve ser sempre garantido. $O$ espaço de soluções viáveis para ambas as tensões foi reduzido a $[0,10]$ - sendo o limite superior correspondente ao limite de estabilidade da malha de realimentação (Cretu et al., 1999) -, com precisão de uma casa decimal.

Na Figura 6 são mostrados os resultados obtidos para dez pares de acelerômetros distintos. São apresentados os valores médios do tempo de convergência, bem como da amplitude das tensões de atuação $V_{1}$ e $V_{2}$, juntamente com seus respectivos valores de desvio padrão - associados à característica não-determinística do algoritmo de evolução diferencial e às características do problema de otimização, uma vez que diferentes combinações de tensões podem resultar em composição da série de Fourier que atende aos requisitos. Além disso, para cada par de acelerômetros, são apresentados os valores da componente fundamental e da soma das demais componentes para o melhor caso.
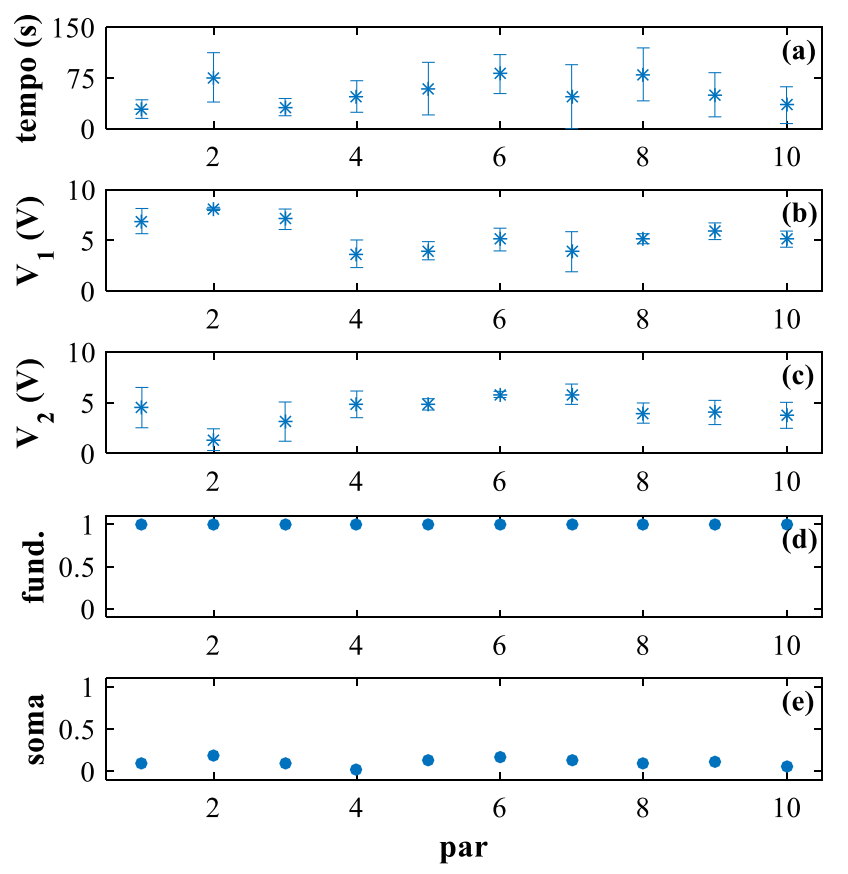

Figura 6. Resultados de calibração de cada par de acelerômetros. Média e desvio padrão do tempo de convergência do algoritmo DE (a). Média e desvio padrão das tensões $V_{1}$ (b) e $V_{2}$ (c). Série de Fourier para o melhor caso - componente fundamental (d) e soma das demais componentes (e).

Analisando-se os resultados da Figura 6, fica claro que, para todos os casos, os critérios das componentes da série de Fourier foram atendidos: a componente fundamental atingiu valor unitário em todos os pares, enquanto a soma das demais componentes da série de Fourier manteve-se abaixo de 0,2. Dessa forma, em termos da composição da série de Fourier do sinal de saída diferencial do sistema, a performance dessa estratégia atingiu os resultados pretendidos. Entretanto, cabe ressaltar que o tempo médio de convergência é relativamente elevado, sendo essa sua principal desvantagem.

O tempo elevado de convergência está relacionado a dois fatores principais: a complexidade da busca pelas variáveis de otimização e o próprio processo de obtenção do espectro. $\mathrm{O}$ primeiro fator associa-se não somente à presença de dois parâmetros de otimização, mas também à sua relação quadrática no modelo dos acelerômetros. Já o segundo fator deve-se à necessidade de levantar o espectro completo novamente para cada combinação das tensões de atuação.

Para validação da calibração realizada para a eliminação do ruído do espectro, utilizou-se um sinal de aceleração externa também dentro da faixa de frequência proposta originalmente, porém distinto daquele utilizado para a calibração. O sinal - com componentes em 70, 110 e $190 \mathrm{~Hz}$, cujas amplitudes são, respectivamente, $27 \mathrm{~m} / \mathrm{s}^{2}, 8,54 \mathrm{~m} / \mathrm{s}^{2}$ e $19,1 \mathrm{~m} / \mathrm{s}^{2}$ - foi aplicado a todos os pares de acelerômetros e as amplitudes das tensões de melhor caso foram usadas para a correção do espectro. Os resultados da coluna à esquerda da Figura 7 mostram os espectros sem a correção da amplitude das tensões, enquanto à direita encontramse os espectros levantados usando as tensões de melhor caso para os pares de acelerômetros 4 e 9 . O par 4 foi escolhido por enquadrar-se nos casos em que, antes da calibração, as diferenças entre os acelerômetros causam algumas distorções no espectro de saída; enquanto isso, o par 9 é um exemplo de um caso extremo das diferenças, levando a espectro completamente poluído antes da calibração proposta, sem possibilidade de distinção entre quaisquer componentes.
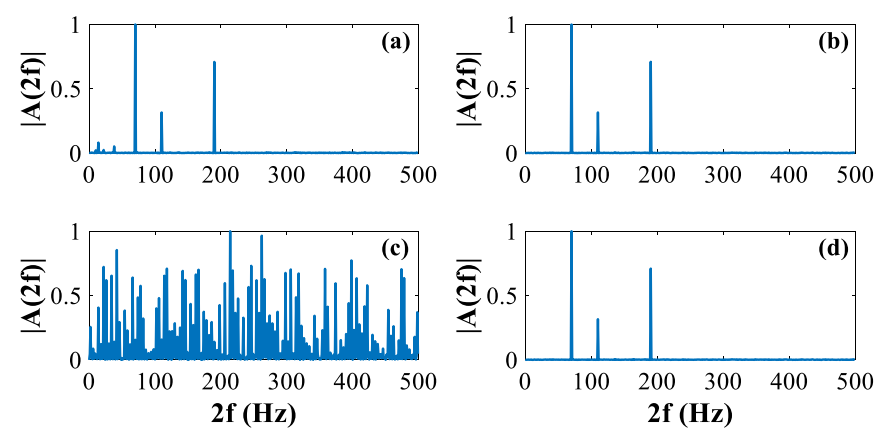

Figura 7. Espectros reais normalizados obtidos com os pares 4 (a) e 9 (c). Espectros normalizados corrigidos obtidos com os pares 4 (b) e 9 (d).

Em ambos os pares, a calibração das tensões de atuação foi capaz de limpar completamente o espectro de vibração, que apresenta apenas as componentes esperadas - e com relações de amplitude adequadas -, mesmo no caso extremo do par 9, que, após a calibração, apresenta apenas duas componentes indesejadas com amplitudes pouco significativas.

\section{CONCLUSÃO}

As simulações realizadas para o analisador de espectro em tempo real evidenciaram a distorção do espectro apresentada quando acelerômetros com parâmetros distintos são utilizados na configuração diferencial. Para contornar esses problemas, a estratégia proposta para a eliminação 
do ruído baseada em evolução diferencial e otimização multiobjetivo mostrou-se adequada, com a calibração proposta sendo capaz de limpar o espectro das acelerações de validação. Embora o ajuste da tensão apresente complexidade e tempo de convergência consideravelmente elevados - devido ao fator quadrático da tensão de atuação e de sua presença na realimentação do modelo do acelerômetro, além do fato de ser uma busca bidimensional pelas variáveis de otimização -, sua vantagem está na eliminação do ruído mesmo em casos extremos. Isso se dá pelo fato da amplitude da tensão usada para a atuação eletrostática relacionar-se com a sensibilidade alcançável e o nível de distorção. Considerando-se, porém, que as estratégias de evolução diferencial são propostas para calibração inicial dos pares, em ambiente controlado, o tempo de processamento dos algoritmos não é fator limitante. A possibilidade de ocorrência desses casos extremos, entretanto, será investigada em trabalhos futuros ao substituir o modelo dos acelerômetros MEMS criado no Matlab por uma simulação de elementos finitos, de forma a se ponderar o benefício do ajuste das tensões de atuação com relação a seu custo computacional.

\section{REFERÊNCIAS}

Bao, M. and Middelhoek, S. (2000). Micro Mechanical Transducers: Pressure Sensors, Accelerometers and Gyroscopes. Handbook of Sensors and Actuators. Elsevier Science.

Beeby, S.P., Tudor, M.J., and White, N.M. (2006). Energy harvesting vibration sources for microsystems applications. Measurement Science and Technology, 17(12), R175.

Betta, G., Liguori, C., Paolillo, A., and Pietrosanto, A. (2002). A dsp-based fft-analyzer for the fault diagnosis of rotating machine based on vibration analysis. IEEE Transactions on Instrumentation and Measurement, 51(6), 1316-1322.

Biefeld, V., Clasbrummel, B., and Binder, J. (2000). Implantable low-g accelerometer for the telemetric monitoring of micro-movements in fracture zones. In 1st Annual International IEEE-EMBS Special Topic Conference on Microtechnologies in Medicine and Biology Proceedings, $497-501$.

Boussaïd, I., Lepagnot, J., and Siarry, P. (2013). A survey on optimization metaheuristics. Information Sciences, 237, 82 - 117. Prediction, Control and Diagnosis using Advanced Neural Computations.

Boyd, S. and Vandenberghe, L. (2004). Convex Optimization. Cambridge University Press, New York, NY, USA.

Cretu, E., Bartek, M., and Wolffenbuttel, R.F. (1999). Analytical modelling for accelerometers with electrically tunable sensitivity. In Technical Proceedings of the 1999 International Conference on Modeling and Simulation of Microsystems, 601-604.

Cretu, E., Rocha, L., and Wolffenbuttel, R. (2003). Electro-mechanical feedback for realization of a mechanical spectrum analyzer. $1407-1410$ vol.2.

Gendreau, M. and Potvin, J.Y. (2010). Handbook of Metaheuristics. Springer Publishing Company, Incorporated, 2nd edition.

Kerr, A. and Mullen, K. (2019). A comparison of genetic algorithms and simulated annealing in maximizing the thermal conductance of harmonic lattices. Computational Materials Science, 157, 31 - 36.

Lee, K.B. and Cho, Y.H. (1998). A triangular electrostatic comb array for micromechanical resonant frequency tuning. Sensors and Actuators A: Physical, 70(1), 112 -117 .

Mezura-Montes, E., Reyes-Sierra, M., and Coello, C.A.C. (2008). Multi-objective Optimization Using Differential Evolution: A Survey of the State-of-the-Art, 173-196. Springer Berlin Heidelberg, Berlin, Heidelberg.

Pedotti, L.A.D.S., Zago, R.M., and Fruett, F. (2017). Fault diagnostics in rotary machines through spectral vibration analysis using low-cost mems devices. IEEE Instrumentation Measurement Magazine, 20(6), 39-44.

Peiner, E., Mikuta, R., Iwert, T., Fritsch, H., Hauptmann, P., Fricke, K., and Schlachetzki, A. (1999). Micromachined resonator for cavitation sensing. Sensors and Actuators A: Physical, 76(1), $266-272$.

Price, K., Storn, R., and Lampinen, J. (2005). Differential Evolution: A Practical Approach to Global Optimization. Natural Computing Series. Springer.

Puers, R., Catrysse, M., Vandevoorde, G., Collier, R., Louridas, E., Burny, F., Donkerwolcke, M., and Moulart, F. (2000). A telemetry system for the detection of hip prosthesis loosening by vibration analysis. Sensors and Actuators A: Physical, 85(1), $42-47$.

Rocha, L.A., Cretu, E., and Wolffenbuttel, R.F. (2005). Mems-based mechanical spectrum analyzer. IEEE Transactions on Instrumentation and Measurement, 54(3), 1260-1265.

Roy, A.L. and Bhattacharyya, T.K. (2015). Design, fabrication and characterization of high performance soi mems piezoresistive accelerometers. Microsystem Technologies, 21(1), 55-63.

Scheibner, D., Mehner, J., Reuter, D., Gessner, T., and Dötzel, W. (2005). A spectral vibration detection system based on tunable micromechanical resonators. Sensors and Actuators A: Physical, 123-124, 63-72. Eurosensors XVIII 2004.

Senturia, S. (2001). Microsystem Design. Kluwer Academic Publishers, US.

Storn, R. and Price, K. (1997). Differential evolution: A simple and efficient heuristic for global optimization over continuous spaces. J. of Global Optimization, 11(4), 341-359.

Tavakkoli, H., Momen, H.G., Sani, E.A., and Yazgi, M. (2017). An inductive mems accelerometer. In 2017 10th International Conference on Electrical and Electronics Engineering (ELECO), 459-463.

Tez, S., Torunbalci, M.M., and Akin, T. (2016). A novel method for fabricating mems three-axis accelerometers using low temperature au-sn eutectic bonding. In 2016 IEEE SENSORS, 1-3.

van Kampen, R. and Wolffenbuttel, R. (1998). Modeling the mechanical behavior of bulk-micromachined silicon accelerometers. Sensors and Actuators A: Physical, $64(2), 137-150$.

Ye, W., Mukherjee, S., and MacDonald, N.C. (1998). Optimal shape design of an electrostatic comb drive in microelectromechanical systems. Journal of Microelectromechanical Systems, 7(1), 16-26.

Younis, M. (2011). MEMS Linear and Nonlinear Statics and Dynamics. Microsystems. Springer US. 